\title{
55. THE VELOCITY DISPERSIONS OF O- AND B-STARS WITHIN A FEW KPC
}

\author{
R. B. SHATSOVA \\ Moscow University, Moscow, U.S.S.R.
}

\begin{abstract}
The results of a study of the dispersion of velocities of O-B5 stars up to a distance of $3 \mathrm{kpc}$ are described.
\end{abstract}

The modern catalogues of motions of OB stars enable us to pass, in the study of stellar kinematics, from the solar vicinity to more distant regions. In their works Rubin and Burley (1964), Feast and Shuttleworth (1965), Petrie (1963), Petrie and Petrie (1968), Bonneau (1967a) and others are already using new observational data for obtaining the velocity field up to $3-4 \mathrm{kpc}$ distance.

To each volume element of the Galaxy one may attribute as kinematic characteristics the velocity of the centroid and the distribution function of peculiar velocities or, what amounts to the same, the velocity dispersion, the asymmetry, etc. In the present paper an attempt is made to derive within the accessible volume of the Galaxy the distribution of velocity dispersions.

As observational basis we used the radial velocities and distances taken from the catalogues of Rubin et al. (1962) and Bonneau (1967b). The volume of space covered by these catalogues was divided into heliocentric rings, or semirings, and sectors. The average number of stars in a single section for $r<3 \mathrm{kpc}$ amounts to 56 and 53 in Rubin's and Bonneau's catalogues respectively.

The observed velocities of stars were corrected for standard solar motion, the Oort's term of galactic rotation and the average residual radial velocity corresponding to the volume element to which the star belongs. A special examination has shown that the average velocity consists of the errors in Oort's terms (mainly due to systematic errors in the distances), the higher order terms of galactic rotation, the radial motion (if any) in the Galaxy, as well as any local motion.

The errors in the mean distances in the Oort's term and in the velocity dispersion were determined by an analytic method which was worked out by us following the ideas of Feast and Shuttleworth (1965). The following values of the parameters of the differential motions were assumed:

$$
\begin{aligned}
& A=15 \mathrm{~km} \mathrm{~s}^{-1} \mathrm{kpc}^{-1}, \quad \omega_{0}^{\prime \prime}=0.7 \mathrm{~km} \mathrm{~s}^{-1} \mathrm{kpc}^{-3}, \\
& \varepsilon_{0}=0.5 \mathrm{~km} \mathrm{~s}^{-1} \mathrm{kpc}^{-1}, \quad \varepsilon_{0}^{\prime}=-0.6 \mathrm{~km} \mathrm{~s}^{-1} \mathrm{kpc}^{-2} .
\end{aligned}
$$

These values figure among other solutions in the paper of Petrie and Petrie (1968). The local motions in the majority of space elements do not exceed the errors of average residual velocities. The maximum velocities appeared in the sections $l^{\mathrm{II}}=$ $120^{\circ}-150^{\circ}$ and $r>2 \mathrm{kpc}\left(V_{r}=7-11 \mathrm{~km} \mathrm{~s}^{-1}\right)$ and $r=1-2 \mathrm{kpc}, R<R_{0}\left(V_{r}=-6 \mathrm{~km} \mathrm{~s}^{-1}\right)$. 
The velocity dispersion, defined by

$$
\sigma^{2}=\frac{1}{n} \sum_{i=1}^{n}\left(V_{i}-\bar{V}\right)^{2},
$$

was corrected for the influence of accidental errors of radial velocities and of distance moduli, as well as for the differential motions of the centroids corresponding to different parts of the section with respect to the centre of the whole section.

The final results, given in Tables I and II, allow us to formulate some inferences:

(1) The dispersions of $V_{r}$ velocities near the sun are approximately the same for the O-B5, O-B1 and B2-B5 spectral groups and are equal to $13-14 \mathrm{~km} \mathrm{~s}^{-1}$.

(2) The dispersion increases with the distance from the sun in the direction of the galactic centre for the bulk of $\mathrm{OB}$ stars, while it is almost constant for the B2-B5 (according to Rubin's catalogue). In the anticentre direction the dispersion either slightly decreases or, within the limits of uncertainty, remains constant.

(3) For $R<R_{0}$ there exists no symmetry with respect to galactic longitude zero.

TABLE I

The dispersion and the parameter $C$ of the peculiar radial velocities of stars from the catalogue of Rubin et al.

\begin{tabular}{|c|c|c|c|c|c|c|c|}
\hline \multirow[b]{2}{*}{ lII } & \multirow{2}{*}{$\begin{array}{l}r \\
\mathrm{kpc}\end{array}$} & \multicolumn{3}{|l|}{ O-B5 } & \multicolumn{3}{|l|}{ B3-B5 } \\
\hline & & $\sigma$ & $C$ & $n$ & $\sigma$ & $C$ & $n$ \\
\hline $330^{\circ}-30^{\circ}$ & $\begin{array}{ll}0 & -0.5 \\
0.5 & -1 \\
1 & -2 \\
2 & -3\end{array}$ & $\begin{array}{l}13.8 \pm 1.7 \\
14.2 \pm 1.3 \\
19.1 \pm 1.4 \\
18.3 \pm 1.8\end{array}$ & $\begin{array}{l}59 \pm 7 \\
63 \pm 6 \\
70 \pm 5 \\
70 \pm 7\end{array}$ & $\begin{array}{l}32 \\
59 \\
96 \\
53\end{array}$ & $\begin{array}{l}14.5 \pm 2.1 \\
11.8 \pm 1.3 \\
14.7 \pm 2.7\end{array}$ & $\begin{array}{l}60 \pm 9 \\
72 \pm 8 \\
68 \pm 12\end{array}$ & $\begin{array}{l}24 \\
42 \\
15\end{array}$ \\
\hline $30^{\circ}-120^{\circ}$ & $\begin{array}{ll}0 & -0.5 \\
0.5 & -1 \\
1 & -2 \\
2 & -3\end{array}$ & $\begin{array}{l}10.0 \pm 0.7 \\
11.1 \pm 0.9 \\
19.1 \pm 1.6 \\
21.3 \pm 2.5\end{array}$ & $\begin{array}{l}48 \pm 3 \\
43 \pm 4 \\
76 \pm 6 \\
74 \pm 9\end{array}$ & $\begin{array}{l}99 \\
71 \\
72 \\
36\end{array}$ & $\begin{array}{r}9.7 \pm 0.8 \\
10.7 \pm 1.7\end{array}$ & $\begin{array}{l}44 \pm 4 \\
49 \pm 8\end{array}$ & $\begin{array}{l}80 \\
19\end{array}$ \\
\hline $120^{\circ}-150^{\circ}$ & $\begin{array}{ll}0 & -0.5 \\
0.5 & -1 \\
1 & -2 \\
2 & -3\end{array}$ & $\begin{array}{r}14.9 \pm 2.4 \\
20.0 \pm 3.0 \\
12.2 \pm 1.7 \\
8.6 \pm 1.1\end{array}$ & $\begin{array}{l}63 \pm 10 \\
93 \pm 14 \\
77 \pm 11 \\
41 \pm 5\end{array}$ & $\begin{array}{l}20 \\
23 \\
26 \\
29\end{array}$ & $\begin{array}{r}14.7 \pm 2.9 \\
10.9 \pm 2.7 \\
5.0 \pm 1.6\end{array}$ & $\begin{array}{l}60 \pm 12 \\
45 \pm 11 \\
33 \pm 10\end{array}$ & $\begin{array}{r}13 \\
8\end{array}$ \\
\hline $150^{\circ}-240^{\circ}$ & $\begin{array}{ll}0 & -0.5 \\
0.5 & -1 \\
1 & -2 \\
2 & -3\end{array}$ & $\begin{array}{l}13.9 \pm 1.1 \\
14.8 \pm 1.5 \\
13.3 \pm 1.0 \\
12.2 \pm 1.8\end{array}$ & $\begin{array}{l}64 \pm 5 \\
59 \pm 6 \\
62 \pm 5 \\
66 \pm 10\end{array}$ & $\begin{array}{l}84 \\
51 \\
91 \\
22\end{array}$ & $\begin{array}{l}13.0 \pm 1.2 \\
11.2 \pm 1.8 \\
11.4 \pm 1.6\end{array}$ & $\begin{array}{l}58 \pm 5 \\
58 \pm 9 \\
93 \pm 13\end{array}$ & $\begin{array}{l}61 \\
20 \\
26\end{array}$ \\
\hline $240^{\circ}-330^{\circ}$ & $\begin{array}{ll}0 & -0.5 \\
0.5 & -1 \\
1 & -2 \\
2 & -3\end{array}$ & $\begin{array}{l}13.4 \pm 0.8 \\
18.7 \pm 2.0 \\
14.6 \pm 1.7 \\
11.4 \pm 1.1\end{array}$ & $\begin{array}{r}56 \pm 4 \\
64 \pm 7 \\
119 \pm 14 \\
53 \pm 5\end{array}$ & $\begin{array}{r}129 \\
45 \\
38 \\
51\end{array}$ & $\begin{array}{r}13.2 \pm 0.9 \\
6.6 \pm 0.9\end{array}$ & $\begin{array}{l}51 \pm 4 \\
61 \pm 8\end{array}$ & $\begin{array}{r}107 \\
26\end{array}$ \\
\hline
\end{tabular}


TABLE II

The dispersion and the parameter $C$ of the peculiar radial velocities of stars from the catalogue of Bonneau

\begin{tabular}{|c|c|c|c|c|c|c|c|}
\hline \multirow[b]{2}{*}{$l^{\mathrm{II}}$} & \multirow{2}{*}{$\begin{array}{l}r \\
\mathrm{kpc}\end{array}$} & \multicolumn{3}{|l|}{ O-B2 } & \multicolumn{3}{|l|}{ B3-B5 } \\
\hline & & $\sigma$ & C & $n$ & $\sigma$ & C & $n$ \\
\hline \multirow[t]{5}{*}{$<120^{\circ}$} & $\begin{array}{ll}0 & -0.5\end{array}$ & $9.3 \pm 1.6$ & $50 \pm 9$ & 17 & $7.6 \pm 0.9$ & $32 \pm 4$ & 35 \\
\hline & $0.5-1$ & $15.5 \pm 1.3$ & $55 \pm 5$ & 67 & $3.2 \pm 0.8$ & $16 \pm 4$ & 9 \\
\hline & $1-2$ & $19.9 \pm 1.6$ & $86 \pm 7$ & 75 & $8.8 \pm 2.1$ & $36 \pm 8$ & 9 \\
\hline & $2-3$ & $18.1 \pm 2.3$ & $89 \pm 11$ & 31 & & & \\
\hline & $3-4$ & $28 \pm 6$ & $130 \pm 28$ & 11 & & & \\
\hline \multirow[t]{5}{*}{$120^{\circ}-180^{\circ}$} & $0-0.5$ & $17.2 \pm 3.0$ & $63 \pm 11$ & 16 & $8.9 \pm 1.2$ & $41 \pm 6$ & 26 \\
\hline & $0.5-1$ & $22.5 \pm 2.7$ & $93 \pm 11$ & 35 & $14.6 \pm 3.0$ & $56 \pm 11$ & 12 \\
\hline & $1-2$ & $12.8 \pm 1.2$ & $89 \pm 9$ & 52 & $12.6 \pm 3.1$ & $119 \pm 30$ & 8 \\
\hline & $2-3$ & $12.6 \pm 1.6$ & $48 \pm 6$ & 32 & & & \\
\hline & $\begin{array}{ll}3 & -4\end{array}$ & $18.0 \pm 4.3$ & $165 \pm 39$ & 9 & & & \\
\hline \multirow[t]{5}{*}{$>180^{\circ}$} & $0-0.5$ & $13.2 \pm 1.5$ & $66 \pm 7$ & 39 & $10.8 \pm 1.1$ & $45 \pm 5$ & 48 \\
\hline & $0.5-1$ & $12.5 \pm 1.5$ & $63 \pm 8$ & 34 & $12.1 \pm 2.5$ & $111 \pm 23$ & 12 \\
\hline & $1-2$ & $10.7 \pm 2.0$ & $79 \pm 8$ & 48 & $4.1 \pm 1.0$ & $20 \pm 5$ & 8 \\
\hline & $2-3$ & $10.7 \pm 2.0$ & $50 \pm 9$ & 15 & & & \\
\hline & $3-4$ & $16.0 \pm 4.0$ & $143 \pm 36$ & 8 & & & \\
\hline
\end{tabular}

(4) In the region of the local spiral arm (in the first quadrant) the dispersion has its minimum value for $r<1 \mathrm{kpc}$ and its maximum value for $r>1 \mathrm{kpc}$. This fact has been established only tentatively, owing to the largeness of the sections of space which contain spiral arms as well as regions between them. It is to be noted that an exclusion of a few stars with $V_{r}>40 \mathrm{~km} \mathrm{~s}^{-1}$ reduces the value of the dispersion to circumsolar values. Small values of $\sigma$ we observe also in the Perseus arm in distinction to the inner arm. Small values in the Orion and Perseus arms are exhibited also by the first absolute moments as may be seen from Petrie's (1963) data.

At the same time the $C$ parameter of the Planck distribution function of peculiar velocities has been considered. The Planck distribution functions for the moduli of space velocities and for their radial components in the simplest form are:

$$
\begin{aligned}
& f(V)=\frac{15}{\pi^{4}} \frac{C^{4}}{V^{5}\left(e^{C / V}-1\right)}, \\
& f\left(V_{r}\right)=\frac{15}{2 \pi^{4} C} \int_{0}^{c / \mid V_{r \mid}} \frac{x^{4} \mathrm{~d} x}{e^{x}-1} .
\end{aligned}
$$

The theoretical curves represent well the observed frequencies in the whole range of velocities, including very large ones, for stars of different types of spectra and luminosity. Theoretical moments of the distributions (2) and (3) truncated at the largest velocity in the sample $V^{*}$, coincide practically with (1). 
In the range of the smallest velocities (for the OB-stars, $V<5 \mathrm{~km} \mathrm{~s}^{-1}$ ) the curve (2) lies lower than the observed one. This defect may be removed by the introduction of a small parameter $\delta$ so that $V^{2} / C^{2}+\delta$ is used as argument instead of $V^{2} / C^{2}$. For small values of $V$ this distribution is transformed into a Maxwellian one, while for large values into the Rayleigh-Jeans distribution.

The dispersion $\sigma$ and the parameter $C$ are connected by the relation

$$
\sigma=S\left(V^{*} / C\right) C
$$

and $S\left(V^{*} / C\right)$ is tabulated by Shatsova $(1965 \mathrm{a}, \mathrm{b})$.

The advantage of $C$, as compared with $\sigma$, consists in its almost full independence of the truncation value. Because of that $C$ may be calculated either from the whole distribution curve of velocities, or from any of its parts, in particular from its 'tail' as well.

The range of the C-values in its main features resembles the range of dispersions. The relative differences of $C$ for different sections are somewhat smaller than for the $\sigma$.

\section{References}

Bonneau, M.: 1967a, Bull. Astron. 2, 13.

Bonneau, M.: 1967b, J. Observateurs 50, 237.

Feast, M. W. and Shuttleworth, M.: 1965, Monthly Notices Roy. Astron. Soc. 130, 245.

Petrie, R. M.: 1963, Publ. Astron. Soc. Pacific 75, 354.

Petrie, R. M. and Petrie, J. K.: 1968, Publ. Dom. Astrophys. Obs. Victoria 13, 253.

Rubin, V. C., Burley, J., Kiasatpoor, A., Klock, B., Pease, G., Ruthscheidt, E. and Smith, C.: 1962 , Astron. J. 67, 491.

Rubin, V. C. and Burley, J.: 1964, Astron. J. 69, 80.

Shatsova, R. B.: 1965a, Astron. Zh. 42, 160.

Shatsova, R. B.: 1965b, Planck's Distribution of Stellar Velocities near the Sun, Rostov-on-the-Don University. 